including low immunogenicity allowing continued dosing, lower reported flare rates and convenient monthly dosing.

Disclosure of Interests: Stephen Smolinski Shareholder of: Selecta Biosciences, Inc., Employee of: Selecta Biosciences, Inc., Alan Kivitz Shareholder of: Novartis, Consultant for: Abbvie, Janssen, Pfizer, UCB, Genzyme, Sanofi, Regeneron, Boehringer Ingelheim, Sun Pharma Advanced Research, Flexion., Paid instructor for: Celgene, Horizon, Merck, Novartis, Pfizer, Genzyme, Sanofi, Regeneron, Speakers bureau: Celgene, Horizon, Merck and Genetech, Flexion, Wesley DeHaan Shareholder of: Selecta Biosciences, Inc., Employee of: Selecta Biosciences, Inc., Lloyd Johnston Shareholder of: Selecta Biosciences, Inc., Employee of: Selecta Biosciences, Inc., Rehan Azeem Shareholder of: Selecta Biosciences, Inc., Employee of: Selecta Biosciences, Inc., Kei Kishimoto Shareholder of: Selecta Biosciences, Inc., Employee of: Selecta Biosciences, Inc.

DOI: 10.1136/annrheumdis-2019-eular.7769

\section{SAT0403 SEX DIFFERENCES ARE PRESENT IN CLINICAL CHARACTERISTICS, BUT NOT IN RESPONSE TO DIFFERENT URATE LOWERING THERAPIES IN PATIENTS WITH GOUT}

Sophie Wanten $^{1}$, Minke Ter Stal ${ }^{1}$, Wing-Yee Kwok ${ }^{2}$, Frank van den Hoogen ${ }^{1,3}$ Marcel Flendrie ${ }^{1}$, Noortje van Herwaarden ${ }^{1,3}$. 'Sint Maartenskliniek, Department of Rheumatology, Nijmegen, Netherlands; ${ }^{2}$ Rijnstate ziekenhuis, Department of Rheumatology, Arnhem, Netherlands; ${ }^{3}$ RadboudUMC, Department of Rheumatology, Nijmegen, Netherlands

Background: Clinical characteristics of gout differ between women and men $^{1}$. Little however is known about the association between these differences and response to treatment. As women seem to have lower mean uric acid excretion compared to $\operatorname{men}^{2,3}$, response to uricosuric agents might be better compared to xanthine oxidase inhibitors.

Objectives: To identify sex differences in clinical characteristics and response to urate lowering therapy (ULT) in patients with gout and in women difference in response to allopurinol or benzbromarone.

Methods: Patients with clinical diagnosis of gout, a first outpatient visit between January 2010 and March 2018 and a follow-up of at least 6 months were included in a retrospective cohort study, with ongoing recruitment, conducted in two rheumatology centres in the Netherlands. From this cohort, patients who started ULT were selected. Clinical characteristics and treatment outcomes of allopurinol and benzbromarone were compared between women and men, including drug survival (corrected for age and renal function) and cumulative incidence of achieving target serum uric acid $(<0.36 \mathrm{mmol} / \mathrm{l})$. In women, difference in cumulative incidence of achieving target serum uric acid while using allopurinol or benzbromarone was compared.

Results: From a total of 519 (105 women/414 men) patients, 513 (104 women/409 men) and 74 (18 women/56 men) patients were included in the allopurinol and/or benzbromarone group, respectively. Clinical characteristics are described in Table 1. Drug survival was similar for women and men for allopurinol (hazard ratio 1.08; 95\% confidence interval $(\mathrm{Cl})$ 0.71-1.64) as well as for benzbromarone (hazard ratio $0.66 ; 95 \% \mathrm{Cl}$ 0.26-1.66) (Figure $1 \mathrm{a}$ and $\mathrm{b}$ ). Cumulative incidences of achieving target serum uric acid were 66\% (69/104) and 73\% (300/ $409)$ after allopurinol and $83 \% \quad(15 / 18)$ and $82 \% \quad(46 / 56)$ after

Table 1. Clinical characteristics

\begin{tabular}{lccc}
\hline & $\begin{array}{c}\text { Women (n } \\
\mathbf{= 1 0 5 )}\end{array}$ & Men (n= 414) & $\begin{array}{c}\text { p- } \\
\text { value }^{*}\end{array}$ \\
\hline Age (years), median (IQR) & $73.9(54.3-71.6)$ & $62.9(54.3-71.6)$ & $<0.01$ \\
Current alcohol use, $\mathrm{n}(\%)$ & $34(45)$ & $275(79)$ & $<0.01$ \\
Comorbidities, $\mathrm{n}(\%)$ & $71(68)$ & $200(48)$ & $<0.01$ \\
Hypertension & $41(39)$ & $76(18)$ & $<0.01$ \\
Renal impairment & $36(34)$ & $80(19)$ & $<0.01$ \\
Diabetes mellitus & & & \\
Diuretics use, $\mathrm{n}(\%)$ & $67(64)$ & $137(33)$ & $<0.01$ \\
Joint involvement, $\mathrm{n}(\%)$ & $14(13)$ & $68(17)$ & 0.38 \\
Monoarthritis & $64(62)$ & $220(62)$ & \\
Oligoarthritis & $26(25)$ & $120(29)$ & \\
Polyarthritis & & & \\
History or presence of tophi, $\mathrm{n}(\%)$ & $39(37)$ & $94(23)$ & $<0.01$ \\
Crystal proven gout, $\mathrm{n}(\%)$ & $89(85)$ & $348(89)$ & 0.86 \\
Baseline serum uric acid (mmol/L), mean & $0.44(0.13)$ & $0.43(0.12)$ & 0.73 \\
(SD) & $(\mathrm{n}=93)$ & $(\mathrm{n}=360)$ & \\
\hline IQR $=$ inter quartile range & & &
\end{tabular}

* P-values for categorical variables were calculated by chi-square analysis, for continuous variables the appropriate (non)parametric analysis was used based on Gaussian distribution. benzbromarone for women and men, respectively. Comparison in women between response to allopurinol and benzbromarone was not statistically significant ( $p=0.15$ ).

Conclusion: Clinical characteristics of gout differ between women and men, confirming previous studies, with women being older, having more comorbidities and using diuretics more often.

Although women have shown to be more uric acid under secretors compared to men, this does not seem to result in a difference in response to a xanthine oxidase inhibitor or an uricosuric agent.

\section{REFERENCES}

[1] Harrold LR, et al. Ann Rheum Dis. 2006 Oct;65(10):1368-72.

[2] Park YB, et al. Clin Rheumatol 2000;19(2):142-6.

[3] te Kampe R. et al. Ann Rheum Dis.2018;77: Suppl A1637

Disclosure of Interests: Sophie Wanten: None declared, Minke ter Stal: None declared, Wing-Yee Kwok: None declared, Frank van den Hoogen: None declared, Marcel Flendrie Grant/research support from: Research grants from Grünenthal, Menarini, Consultant for: Yes, advisory board for Grünenthal in 2017, Paid instructor for: Yes, for Menarini, Noortje van Herwaarden: None declared

A
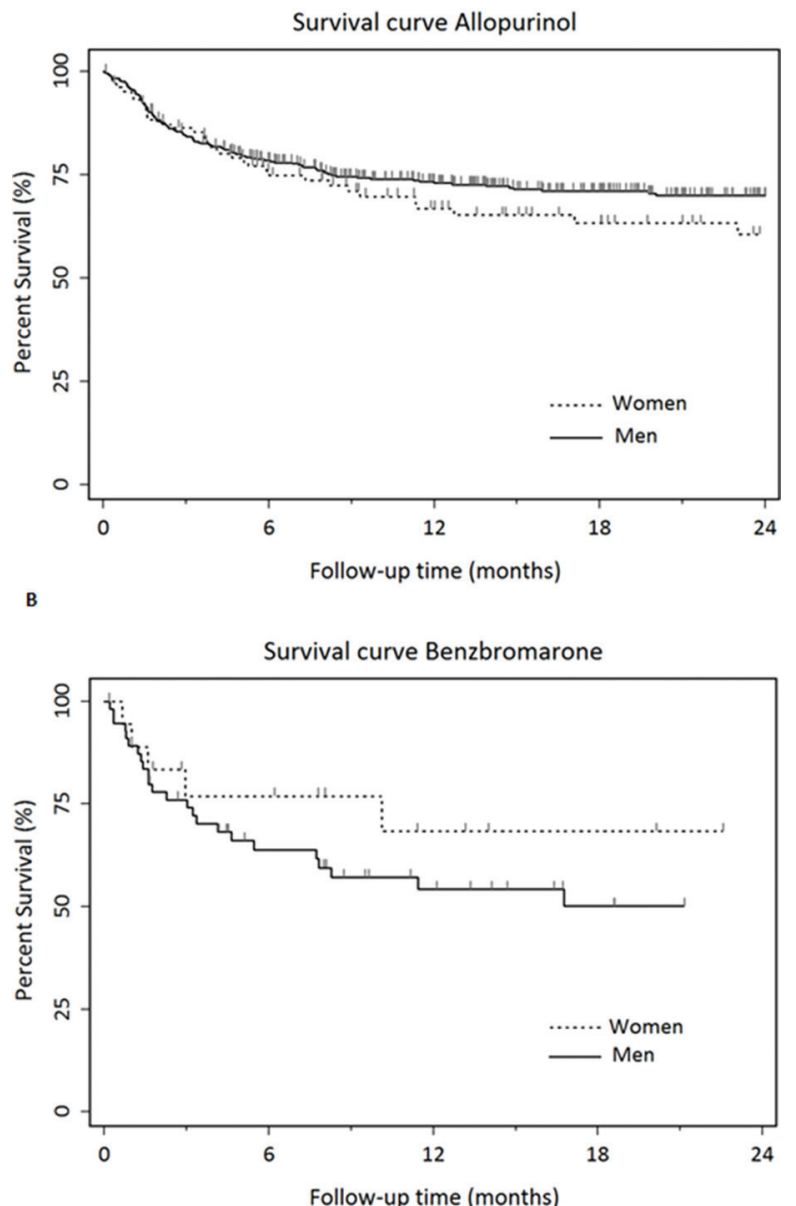

DOI: 10.1136/annrheumdis-2019-eular.930

\section{SAT0404 PRETREATMENT AND CO-ADMINISTRATION WITH METHOTREXATE IMPROVED DURABILITY OF PEGLOTICASE RESPONSE: A PROSPECTIVE, OBSERVATIONAL, PROOF-OF-CONCEPT, CASE SERIES}

John Botson ${ }^{1}$, Jeff Peterson ${ }^{2} .{ }^{1}$ Orthopedic Physicians Alaska, Anchorage, United States of America; ${ }^{2}$ Western Washington Arthritis Clinic, Bothell, United States of America

Background: Pegloticase is a recombinant DNA-produced porcine-like uricase enzyme, which metabolizes relatively insoluble urate to highly soluble allantoin. It is used in the treatment of refractory gout which has 